\title{
Studies on Metal Carbonate Equilibria. 23. Complex Formation in the Th(IV)- $\mathrm{H}_{2} \mathrm{O}-\mathrm{CO}_{2}(\mathrm{~g})$ System
}

\author{
Ingmar Grenthe ${ }^{\star}$ and Bo Lagerman ${ }^{\dagger}$ \\ Department of Inorganic Chemistry, Royal Institute of Technology, S-100 44 Stockholm, Sweden
}

\begin{abstract}
Grenthe, I. and Lagerman, B., 1991. Studies on Metal Carbonate Equilibria. 23. Complex Formation in the Th(IV)- $\mathrm{H}_{2} \mathrm{O}-\mathrm{CO}_{2}(\mathrm{~g})$ System. - Acta Chem. Scand. 45: 231-238.

The stoichiometry and the stability of the complexes formed in the Th(IV)- $\mathrm{H}_{2} \mathrm{O}-\mathrm{CO}_{2}(\mathrm{~g})$ system have been investigated by the EMF technique in a $3.00 \mathrm{M} \mathrm{NaClO}$ ionic medium at $25^{\circ} \mathrm{C}$, using coulometry to vary the hydrogen ion concentration. The following concentration ranges have been investigated: $0.2 \times 10^{-3}<[\mathrm{Th}]_{\mathrm{tot}}<6 \times 10^{-3}$; $2.2 \leq-\log \left[\mathrm{H}^{+}\right] \leq 4.5 ; 0 \leq P_{\mathrm{CO}_{2}} \leq 0.97$. The chemical model selected for the hydroxide system consists of two mononuclear complexes, $\mathrm{ThOH}^{3+}\left(\log { }^{*} \beta_{1}=\right.$ $-4.35 \pm 0.09)$ and $\operatorname{Th}(\mathrm{OH})_{4}(\mathrm{aq}) \quad\left(\log { }^{*} \beta_{4}=-16.65 \pm 0.04\right)$, two binuclear complexes, $\mathrm{Th}_{2}(\mathrm{OH})_{2}{ }^{6+}\left(\log { }^{*} \beta_{2,2}=-5.10 \pm 0.17\right)$ and $\mathrm{Th}_{2}(\mathrm{OH})_{3}{ }^{5+}\left(\log { }^{*} \beta_{2,3}=\right.$ $-7.87 \pm 0.05)$, two tetranuclear complexes, $\mathrm{Th}_{4}(\mathrm{OH})_{8}{ }^{8+}\left(\log { }^{*} \beta_{4,8}=-19.6 \pm 0.2\right)$ and $\mathrm{Th}_{4}(\mathrm{OH})_{12}{ }^{4+}\left(\log { }^{*} \beta_{4,12}=-34.86 \pm 0.05\right)$, and two hexanuclear complexes, $\mathrm{Th}_{6}(\mathrm{OH})_{14}{ }^{10+}\left(\log * \beta_{6,14}=-33.67 \pm 0.05\right)$ and $\mathrm{Th}_{6}(\mathrm{OH})_{16}{ }^{8+}\left(\log * \beta_{6,16}=-42.90 \pm 0.4\right)$; the last is present in very small amounts. The $(4,12)$ complex is assumed to have the same basic structure as the $(4,8)$ complex, but with four terminal $\mathrm{OH}$ groups, one on each Th(IV).

Addition of $\mathrm{CO}_{2}(\mathrm{~g})$ to the $\mathrm{Th}(\mathrm{IV})-\mathrm{H}_{2} \mathrm{O}$ system in the $-\log \left[\mathrm{H}^{+}\right]$range explored results in very steep formation curves which depend on $P_{\mathrm{CO}_{2}},[\mathrm{Th}]_{\mathrm{tot}}$ and $-\log \left[\mathrm{H}^{+}\right]$. It is not possible to identify a unique chemical model for the carbonate system, only to conclude that ternary complexes of high nuclearity are formed. The experimental data at the lowest Th(IV) concentrations can approximately be described with species of the type $\mathrm{Th}_{16}(\mathrm{OH})_{52}\left(\mathrm{CO}_{2}\right)_{16}{ }^{12+}$ and $\mathrm{Th}_{8}(\mathrm{OH})_{24}\left(\mathrm{CO}_{2}\right)_{2}{ }^{8+}$. At somewhat higher [Th $]_{\text {tot }}$ a precipitate is formed, however, not in the test solutions used to deduce the chemical model(s).

An exploratory spectrophotometric study of the $\mathrm{U}(\mathrm{IV})-\mathrm{H}_{2} \mathrm{O}-\mathrm{CO}_{2}(\mathrm{~g})$ system at $-\log \left[\mathrm{H}^{+}\right] \approx 2$ showed a pronounced increase in the intensity of the U(IV) absorption band centred around $650 \mathrm{~m}$, also indicating the binding of $\mathrm{CO}_{2}$. It seems likely that the speciation of thorium and other tetravalent actinides, and hence their solubility, will also be influenced by the presence of $\mathrm{CO}_{2} / \mathrm{HCO}_{3}{ }^{-}$in the acid ranges.
\end{abstract}

Hydroxide and carbonate are two of the most important ligands in ground-water systems. The $\mathrm{pH}$ and the partial pressure of $\mathrm{CO}_{2}(\mathrm{~g})$ are decisive parameters for the solubility of minerals and sparingly soluble metal oxides/hydroxides. Accordingly, the mobility of many elements in nature, usually in trace concentrations, is also strongly influenced by $\mathrm{pH}$ and $P_{\mathrm{CO}_{2}}$. In previous parts of this series we have studied the complex formation of lanthanide and actinide elements in the $\mathrm{H}_{2} \mathrm{O}-\mathrm{CO}_{2}(\mathrm{~g})$ system, mainly in order to determine stoichiometries and to discuss the structures of the complexes formed. There are a number of experimental complications that have made it difficult to obtain chemical information about these systems; a typical example of the problems encountered will be presented in this study.

Experimental investigations of actinide(IV)- $\mathrm{H}_{2} \mathrm{O}-\mathrm{CO}_{2}(\mathrm{~g})$ systems have been made in bicarbonate/carbonate solutions, usually in the concentration range in which mono-

\footnotetext{
* To whom correspondence should be addressed.

${ }^{\dagger}$ This is a part of the Ph.D. diss. of B.L. at the Royal Institute of Technology, S-100 44 Stockholm, Sweden.
}

nuclear $\mathrm{M}\left(\mathrm{CO}_{3}\right)_{5}{ }^{6-}$ and $\mathrm{M}\left(\mathrm{CO}_{3}\right)_{4}{ }^{4-}$ are formed. ${ }^{1,2}$ Measurements at lower carbonate concentrations are complicated by the extremely low solubility of the M(IV) hydrous oxides. ${ }^{1}$ No quantitative information about the formation of carbonate/bicarbonate containing M(IV) species in acid solutions were known until our previous preliminary study of the $\mathrm{Th}(\mathrm{IV})-\mathrm{H}_{2} \mathrm{O}-\mathrm{CO}_{2}(\mathrm{~g})$ system. ${ }^{3}$ This study indicated clearly that the titration curves were strongly influenced by $\mathrm{CO}_{2}(\mathrm{~g})$, even at $-\log \left[\mathrm{H}^{+}\right]$around 3 , where the hydrolysis of Th(IV) is negligible. We have extended the previous investigation in order to try to obtain a better chemical model of the system. In order to achieve this it was first necessary to study the two-component system Th(IV)- $\mathrm{H}_{2} \mathrm{O}$ to obtain good hydrolysis data. There are a very large number of previous experimental studies; reviews and discussions of these data have been given by Baes and Mesmer, ${ }^{4}$ and more recently by Brown et al. ${ }^{5}$ Baes and Mesmer discuss the difficulty of obtaining a unique chemical model, indicated for example by the fact that the two sets $\{(2,2),(4,8),(6,15)\}$ and $\{(2,2),(2,3),(6,14)\}$ fit the experimental data equally well. The numbers within parentheses refer to the stoichiometric coefficients $p$ and $q$ in the 
formula $\mathrm{Th}_{p}(\mathrm{OH})_{q}^{4 p-q}$. Brown et al. ${ }^{5}$ propose the formation of a $(4,12)$ complex, which is predominant in the range $3.5<-\log \left[\mathrm{H}^{+}\right]<4$.

There is some structural evidence to support the proposed stoichiometries. The $(2,2)$ complex has been found as a discrete complex in the solid state ${ }^{6}{ }^{6}$ while the structures of the hydroxo complexes $(2,2)$ and $(6,14)$ in solution have been discussed by Magini et al. ${ }^{7}$ A problem with the largeangle $\mathrm{X}$-ray diffraction studies of solutions is the very high thorium concentrations that have to be used. Both LAXS and light-scattering data ${ }^{8}$ indicate an average degree of condensation that does not exceed 5 or 6 at $Z=2.4$, where $Z$ is the average number of bonded $\mathrm{OH}^{-}$per Th(IV).

Previous experimental data have been obtained by titrations with hydroxide or $\mathrm{NaHCO}_{3}{ }^{5}$ This may cause local precipitation of thorium hydroxide, or the formation of carbonate complexes, cf. Ref. 9. In order to avoid this difficulty we have studied solutions with low total concentrations of thorium by using EMF measurements in combination with coulometric generation of $\mathrm{H}^{+}$. The corresponding three-component system was studied in the same way, now in the presence of $\mathrm{CO}_{2}(\mathrm{~g})$ of known partial pressure. The experimental technique and the method used to analyze the data have been described previously. ${ }^{4}$

We have investigated the following concentration ranges: $0.2 \times 10^{-3}<[\mathrm{Th}]_{\text {tot }}<6 \times 10^{-3} \mathrm{M} ; 2.5 \leq-\log \left[\mathrm{H}^{+}\right] \leq 5 ; 0 \leq$ $P_{\mathrm{CO}_{2}} \leq 0.97$ atm, all in a $3.00 \mathrm{M} \mathrm{NaClO}_{4}$ ionic medium.

\section{Experimental}

Chemicals. Thorium perchlorate solutions were prepared by precipitation of $\mathrm{Th}(\mathrm{OH})_{4}(\mathrm{~s})$ with $\mathrm{NH}_{4} \mathrm{OH}(\mathrm{aq})$ (Merck p.a.) from a solution of $\mathrm{Th}\left(\mathrm{NO}_{3}\right)_{4}$ (Merck p.a.); the precipitate was carefully washed with bidistilled water and then dissolved with $\mathrm{HClO}_{4}$ (Merck p.a.). The concentration of Th was analyzed gravimetrically as $\mathrm{ThO}_{2}{ }^{10}$ The excess concentration of $\mathrm{H}^{+}$in the thorium solutions was determined by the Gran method. ${ }^{11}$ The $\mathrm{CO}_{2}(\mathrm{~g})-\mathrm{N}_{2}(\mathrm{~g})$ gas mixtures used in the experiments were prepared and analyzed by AGA. These gas mixtures were purified and conditioned by passage through $10 \% \mathrm{H}_{2} \mathrm{SO}_{4}$ and $3 \mathrm{M} \mathrm{NaClO}_{4}$ solutions, prior to passage through the test solution. The $\mathrm{N}_{2}$ gas (AGA) used in the hydrolysis experiments was purified and conditioned by passing it through $10 \% \mathrm{H}_{2} \mathrm{SO}_{4}, 10 \%$ $\mathrm{NaOH}$ and $3 \mathrm{M} \mathrm{NaClO}_{4}$ solutions, respectively.

Method. The chemical model is based on potentiometric determinations of the concentration of free $\mathrm{H}^{+}$in solutions of known total concentrations of Th(IV) and known total acidity. The latter quantity was varied coulometrically. The Th(IV) - hydroxide system was first studied in each titration series by coulometric generation of hydroxide. ${ }^{*}$ Then, at the highest value of $-\log \left[\mathrm{H}^{+}\right], \mathrm{CO}_{2}$ was passed through the system and the titration was reversed by coulometric addition of protons; in some cases the titration was con-

\footnotetext{
${ }^{*}$ The experimental data are given in Table 1 .
}

tinued in the basic direction. in some titrations $\mathrm{CO}_{2}(\mathrm{~g})$ was introduced at the lowest value of $-\log \left[\mathrm{H}^{+}\right]$, which was then increased by coulometric generation of hydroxide. The same result was obtained (within $1 \mathrm{mV}$ ) independently of the direction of the $-\log \left[\mathrm{H}^{+}\right]$change. The stability of the measured potential was excellent; it remained constant and was reproducible within $0.05 \mathrm{mV}$ when measured over $4 \mathrm{~h}$. No precipitation could be observed in the concentration ranges studied. ${ }^{\dagger}$ We also made an attempt to detect the formation of a precipitate at the highest $Z$ values in the carbonate system. This was done by using dynamic lightscattering data. However, the results were not conclusive: one sample indicated the presence of colloids ca. $1000 \AA$ in size, the other gave no indications. The experiments were carried out by Dr. Marco Caceci at CEA, Fontenay-auxRoses, France, and transportation of the samples might have caused the precipitation, e.g. through a decrease in the partial pressure of $\mathrm{CO}_{2}$. Measurements of this type should probably be made on line with the titration experiment.

In a separate experiment we made a spectrophotometric study of the $\mathrm{U}(\mathrm{IV})-\mathrm{H}_{2} \mathrm{O}-\mathrm{CO}_{2}$ system in order to obtain information on the possible binding of carbonate in acid solutions. The experiment was carried out using the method described by Bruno et al. ${ }^{2}$ At $-\log \left[\mathrm{H}^{+}\right]=1.8$, the absorption band at about $650 \mathrm{~nm}$ increased about $40 \%$ in intensity when $\mathrm{CO}_{2}$ was bubbled through the solution, but the positions of maximum absorbance did not change significantly. After a few hours a black precipitate was formed. However, there was no indication that the initial intensity change was due to the presence of colloidal particles.

Analysis and refinement of the hydroxide data. The various chemical models were tested by using the LETAGROP (Ref. 12 and references therein) least-squares method with the measured EMF as the error-carrying variable. This is the most reasonable choice in a coulometric titration for which the error in the titration procedure is very small. The chemical models have been based on the previously available information on the hydrolysis of Th(IV). All models we have tested so far include a set of mono-, bi-, tetra- and hexanuclear species as indicated in Table 3. Based on the various refinements we have selected model 1 as the "best" one, because it gives a significantly lower error-square sum than the other models $\left[U=17.27(\mathrm{mV})^{2}\right]$. Model 6, with an error-square sum of $23.86(\mathrm{mV})^{2}$, can be rejected, as can models 3,4 and 5 , which do not contain the $(4,12)$ complex. These models have $U$-values around 25 . The difference between models 1 and 6 is the inclusion of the $(2,3)$ and $(6,16)$ complexes in the former model; this results in a significant decrease in the error-square sum. All the models contain a large number of coexisting species, resulting in correlations between the equilibrium constants and difficulties in obtaining a unique chemical model. The $(4,12)$ and

\footnotetext{
${ }^{\dagger}$ The experimental data are given in Table 2.
} 
Table 1. Experimental Th(IV) $-\mathrm{H}_{2} \mathrm{O}$ data given as log $\left[\mathrm{H}^{+}\right], H_{\text {tot }} / 10^{3} \mathrm{~mol} \mathrm{~kg}{ }^{-1} \cdot H_{\text {tot }}=\left[\mathrm{H}^{+}\right]-\left[\mathrm{OH}^{-}\right]-\Sigma q\left[(\mathrm{Th})_{p}(\mathrm{OH})_{q}\right]($ total analytical acidity of the solution).

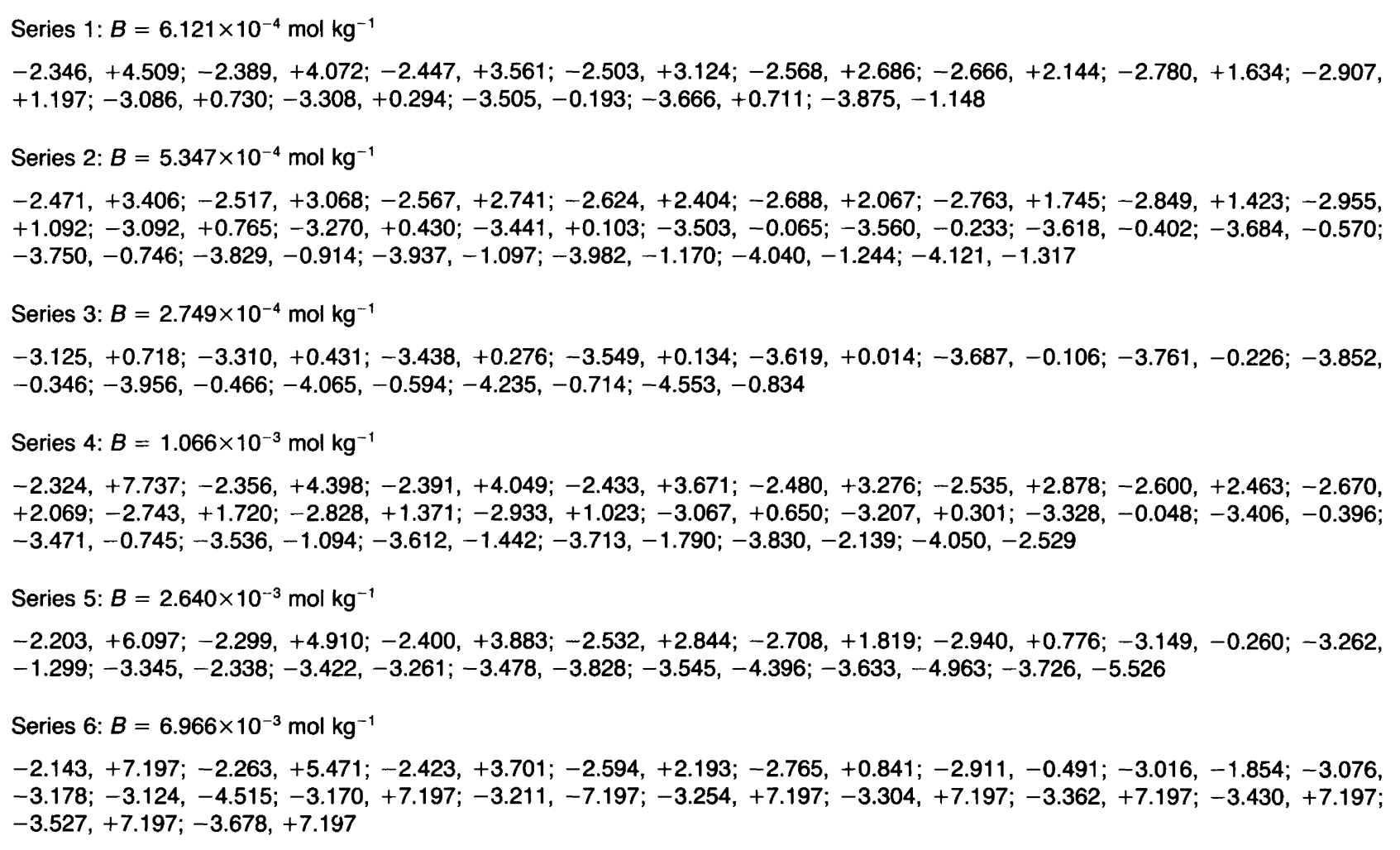

$(6,18)$ complexes are formed in the same $-\log \left[\mathrm{H}^{+}\right]$region; however, the agreement between the model and the experiments in this region is distinctly better with the $(4,12)$ complex. Some of the species also occur in fairly small concentrations. Within each group of models one cannot distinguish between models containing minor species and those where these are absent. If $(6,16)$ is removed from model 1 the $U$-value increases to $17.78 \mathrm{mV}^{2}$; if $(1,3)$ is also removed we obtain $U=18.89 \mathrm{mV}^{2}$. The values of the other equilibrium constants and their uncertainties do not change significantly in this process. Further discussions of the selected chemical model will be given in the next section. In Fig. 1 we have plotted the experimental $Z\left(-\log \left[\mathrm{H}^{+}\right]\right)$data and indicated the fit between the experimental data and the selected model with the full curves.

Analysis of the carbonate data. By working at a constant partial pressure of $\mathrm{CO}_{2}(\mathrm{~g})$ in the various titrations we can reduce the three-component problem to a two-component problem. The shape of the curves (cf. Fig. 2) demonstrates very clearly that $\mathrm{CO}_{2}$ has a pronounced effect on the system. The $Z\left(-\log \left[\left[\mathrm{H}^{+}\right]\right)\right.$curves [where $Z$ is the average number of hydroxide groups per Th(IV)] are much steeper, indicating the formation of only one or two predominant complexes with a higher value of $Z$ at a given value of $\log \left[\mathrm{H}^{+}\right]$than in the binary $\mathrm{Th}(\mathrm{IV})-\mathrm{H}_{2} \mathrm{O}$ system. $Z$ also varies with $P_{\mathrm{CO}_{2}}$ and the total concentration of Th(IV). It is not possible to describe the whole set of $[\mathrm{Th}]_{\mathrm{tot}},-\log \left[\mathrm{H}^{+}\right]$, $P_{\mathrm{CO}_{2}}$ values with one model. For each metal ion concentration the $-\log \left[\mathrm{H}^{+}\right], P_{\mathrm{CO}_{2}}$ data can be described with two complexes. However, this set cannot be used to describe the data at other $[\mathrm{Th}]_{\text {tot }}$ values. An example is shown in Fig. 2 for the two lowest metal ion concentrations, using $\mathrm{Th}_{16}(\mathrm{OH})_{52}\left(\mathrm{CO}_{2}\right)_{16}{ }^{12+}$ and $\mathrm{Th}_{8}(\mathrm{OH})^{24}\left(\mathrm{CO}_{2}\right)_{2}{ }^{8+}$ as the model. However, this stoichiometry is by no means unique, and we have not found it possible to find a unique chemical model for the system based on the present experimental information.

The chemically important result is instead that we demonstrate how $\mathrm{CO}_{2}(\mathrm{~g})$ can bring about the formation of aggregates of very high nuclearity in the system. One might ask if the experimental findings are not a result of precipitation of a sparingly soluble thorium carbonate phase. Visual observation did not indicate the formation of solids, the measured EMF values were also quite constant over long periods of time, indicating constant chemical conditions in the test solutions. The dynamic light-scattering experiments did not give us any definitive evidence for the formation of colloidal precipitates in the plateau region.

There are literature reports ${ }^{13}$ on solids of the type $\mathrm{ThOCO}_{3} \cdot n \mathrm{H}_{2} \mathrm{O} \quad(n=1,2,3,7)$ and basic carbonates, $\mathrm{ThO}_{2} \cdot \mathrm{ThOCO}_{3} \cdot 0.5 \mathrm{H}_{2} \mathrm{O}$, but no quantitative information on structure or solubilities is available. 
Table 2. Experimental Th(IV)- $\mathrm{H}_{2} \mathrm{O}-\mathrm{CO}_{2}(\mathrm{~g})$ data given as $\log \left[\mathrm{H}^{+}\right], H_{\mathrm{tot}} / 10^{3} \mathrm{~mol} \mathrm{~kg}{ }^{-1}, P_{\mathrm{CO}_{2}} / \mathrm{atm}$.

$H_{\text {tot }}=\left[\mathrm{H}^{+}\right]-\left[\mathrm{OH}^{-}\right]-\Sigma q\left[(\mathrm{Th})_{p}(\mathrm{OH})_{q}\left(\mathrm{CO}_{2}\right)_{r}\right]$ (total analytical acidity of the solution).

Series 1: $[\mathrm{Th}(\mathrm{IV})]_{\mathrm{tot}}=2.758 \times 10^{-4} \mathrm{~mol} \mathrm{~kg}^{-1}$

$-2.922,+1.191,+0.293 ;-2.995,+0.998,+0.293 ;-3.062,+0.843,+0.293 ;-3.142,+0.688,+0.293 ;-3.233,+0.533,+0.293$. $-3.342,+0.378,+0.293 ;-3.401,+0.301,+0.293 ;-3.487,+0.147,+0.293 ;-3.571,-0.164,+0.293 ;-3.658,-0.381,+0.293 ;$ $-3.705,-0.462,+0.293 ;-3.777,-0.552,+0.292 ;-3.878,-0.633,+0.292 ;-4.029,-0.712,+0.292 ;-4.216,-0.788,+0.292 ;$

$-4.415,-0.865,+0.292 ;-4.586,-0.942,+0.291 ;-4.720,-1.018,+0.291 ;-4.829,-1.095,+0.291 ;-4.920,-1.171,+0.291$;

$-4.996,-1.247,+0.291$

Series 2: $[\mathrm{Th}(\mathrm{IV})]_{\mathrm{tot}}=2.758 \times 10^{-4} \mathrm{~mol} \mathrm{~kg}^{-1}$

$-2.999,+0.973,+0.973 ;-3.037,+0.879,+0.973 ;-3.079,+0.786,+0.973 ;-3.146,+0.650,+0.974 ;-3.229,+0.496,+0.973 ;$ $-3.316,+0.343,+0.973 ;-3.367,+0.189,+0.973 ;-3.393,+0.036,+0.974 ;-3.429,-0.117,+0.974 ;-3.472,-0.270,+0.972 ;$ $-3.532,-0.422,+0.973 ;-3.620,-0.570,+0.972 ;-3.680,-0.642,+0.972 ;-3.827,-0.773,+0.972 ;-4.029,-0.904,+0.972 ;$

$-4.194,-1.014,+0.972 ;-1.337,-1.125,+0.972 ;-4.449,-1.230,+0.972 ;-4.734,-1.627,+0.972$

Series 3: $[\mathrm{Th}(\mathrm{IV})]_{\mathrm{tot}}=6.121 \times 10^{-4} \mathrm{~mol} \mathrm{~kg}^{-1}$

$-5.957,-5.760,+0.287 ;-5.925,-5.490,+0.286 ;-5.896,-5.258,+0.286 ;-5.864,-5.031,+0.286 ;-5.830,-4.803,+0.286 ;$ $-5.794,-4.575,+0.286 ;-5.754,-4.347,+0.286 ;-5.710,-4.119,+0.286 ;-5.662,-3.892,+0.286 ;-5.607,-3.664,+0.286 ;$ $-5.546,-3.436,+0.286 ;-5.475,-3.208,+0.286 ;-5.391,-2.980,+0.286 ;-5.288,-2.751,+0.286 ;-5.156,-2.523,+0.286 ;$ $-4.963,-2.295,+0.286 ;-4.702,-2.066,+0.287 ;-3.817,-1.609,+0.287 ;-3.627,-1.380,+0.288 ;-3.525,-1.166,+0.288 ;$ $-3.468,-0.937,+0.290 ;-3.419,-0.723,+0.290 ;-3.380,-0.485,+0.290 ;-3.355,-0.270,+0.292 ;-3.319,-0.027,+0.292 ;$ $-3.264,+0.226,+0.287 ;-3.193,+0.468,+0.287$

Series 4: $[\mathrm{Th}(\mathrm{IV})]_{\mathrm{tot}}=6.121 \times 10^{-4} \mathrm{~mol} \mathrm{~kg}^{-1}$

$-2.570,+2.683,+0.977 ;-2.687,+2.068,+0.979 ;-2.839,+1.426,+0.980 ;-3.032,+0.816,+0.980 ;-3.214,+0.382,+0.979 ;$ $-3.263,+0.078,+0.977 ;-3.284,-0.221,+0.976 ;-3.308,-0.514,+0.973 ;-3.348,-0.835,+0.970 ;-3.398,-1.132,+0.968 ;$ $-3.477,-1.447,+0.969 ;-3.613,-1.736,+0.971 ;-3.934,-2.024,+0.972 ;-4.328,-2.313,+0.972 ;-4.584,-2.601,+0.973 ;$ $-4.756,-2.889,+0.973 ;-4.882,-3.177,+0.974 ;-4.996,-3.513,+0.974 ;-5.084,-3.833,+0.974 ;-5.157,-4.153,+0.974 ;$ $-5.216,-4.446,+0.974 ;-5.267,-4.739,+0.974 ;-5.315,-5.045,+0.974 ;-5.395,-5.632,+0.974 ;-5.522,-6.795,+0.974 ;$ $-5.580,-7.445,+0.972 ;-5.628,-8.053,+0.972 ;-5.668,-8.630,+0.972 ;-5.706,-9.207,+0.972 ;-5.732,-9.635,+0.972 ;$

$-5.764,-10.213,+0.972 ;-5.796,-10.799,+0.971 ;-5.824,-11.387,+0.971 ;-5.850,-11.961,+0.971$

Series 5: $[\mathrm{Th}(\mathrm{IV})]_{\mathrm{tot}}=2.641 \times 10^{-3} \mathrm{~mol} \mathrm{~kg}^{-1}$

$-2.763,+1.456,+0.290 ;-2.830,+1.113,+0.290 ;-2.891,+0.806,+0.290 ;-2.959,+0.468,+0.290 ;-3.023,+0.130,+0.290 ;$ $-3.083,-0.207,+0.290 ;-3.135,-0.545,+0.290 ;-3.181,-0.883,+0.290 ;-3.213,-1.220,+0.290 ;-3.225,-1.558,+0.290 ;$ $-3.223,-1.956,+0.291 ;-3.233,-2.395,+0.291 ;-3.241,-2.848,+0.291 ;-3.250,-3.303,+0.291 ;-3.258,-3.725,+0.291 ;$ $-3.269,-4.153,+0.290 ;-3.277,-4.613,+0.290 ;-3.288,-5.073,+0.290 ;-3.307,-5.562,+0.289 ;-3.312,-5.991,+0.289$; $-3.332,-6.496,+0.289 ;-3.350,-6.931,+0.289 ;-3.383,-7.420,+0.289 ;-3.429,-7.883,+0.289 ;-3.484,-8.360,+0.291 ;$ $-3.570,-8.814,+0.292 ;-3.625,-9.028,+0.293 ;-3.953,-9.480,+0.294 ;-4.522,-9.936,+0.294 ;-4.876,-10.363,+0.294 ;$ $-5.131,-10.817,+0.294$

Series 6: $[\mathrm{Th}(\mathrm{IV})]_{\mathrm{lot}}=2.641 \times 10^{-3} \mathrm{~mol} \mathrm{~kg}^{-1}$

$-2.732,+1.653,+0.960 ;-2.840,+1.011,+0.960 ;-2.946,+0.341,+0.961 ;-2.998,+0.045,+0.964 ;-3.020,-0.739,+0.965 ;$ $-3.038,-1.328,+0.964 ;-3.056,-1.894,+0.963 ;-3.065,-2.573,+0.954 ;-3.068,-3.310,+0.953 ;-3.092,-4.048,+0.953 ;$ $-3.113,-4.729,+0.956 ;-3.123,-5.382,+0.958 ;-3.158,-6.065,+0.958 ;-3.202,-8.479,+0.969 ;-3.252,-9.247,+0.969 ;$ $-3.316,-10.015,+0.969 ;-3.439,-10.782,+0.969 ;-3.833,-11.549,+0.969 ;-4.475,-12,315,+0.969 ;-4.822,-13.082,+0.969$; $-5.034,-13.850,+0.969$

Series 7: $[\mathrm{Th}(\mathrm{IV})]_{\mathrm{lot}}=6.966 \times 10^{-3} \mathrm{~mol} \mathrm{~kg}^{-1}$

$-2.303,+4.873,+0.286 ;-2.367,+4.118,+0.286 ;-2.440,+3.347,+0.286 ;-2.508,+2.659,+0.286 ;-2.590,+1.887,+0.286 ;$ $-2.663,+1.199,+0.286 ;-2.743,+0.427,+0.286 ;-2.818,-0.346,+0.286 ;-2.877,-1.032,+0.289 ;-2.968,-2.142,+0.289 ;$ $-3.038,-3.274,+0.287 ;-3.088,-4.520,+0.287 ;-3.119,-6.943,+0.286 ;-3.133,-10.075,+0.286 ;-3.158,-13.266,+0.289 ;$

$-3.164,-14.821,+0.289 ;-3.182,-16.290,+0.288 ;-3.196,-17.488,+0.288 ;-3.212,-18.742 ;-3.230,-19.857,+0.286$

Series 8: $[\mathrm{Th}(\mathrm{IV})]_{\mathrm{lot}}=6.966 \times 10^{-3} \mathrm{~mol} \mathrm{~kg}^{-1}$

$-2.548,+2.171,+0.970 ;-2.677,+0.784,+0.970 ;-2.804,-0.773,+0.970 ;-2.885,-2.000,+0.969 ;-2.929,-2.812,+0.969 ;$ $-2.945,-4.909,+0.971 ;-2.956,-7.314,+0.971 ;-2.972,-9.269,+0.970 ;-2.989,-12.291,+0.970 ;-3.005,-14.365,+0.973 ;$ $-3.022,-15.785,+0.976 ;-3.037 ;-17.115,+0.976$ 
Table 3. $-\log { }^{*} \beta_{\rho q} \pm 3 \sigma$ values and standard deviation in the error-carrying variable $E$ (in $\mathrm{mV}$ ) [ $\left.\sigma(E M F)\right]$ and the error-square sum $U$ are given for the least-squares analysis of different chemical models using the hydrolysis data from the present study.

\begin{tabular}{lcccccc}
\hline$p, q$ & Model 1 & Model 2 & Model 3 & Model 4 & Model 5 & Model 6 \\
\hline 1,1 & $4.35 \pm 0.09$ & $4.06 \pm 0.07$ & $4.12 \pm 0.07$ & $4.9 \pm 0.3$ & $4.8 \pm 0.2$ & $4.08 \pm 0.06$ \\
1,2 & - & - & - & $8.3 \pm 0.2$ & - & - \\
1,3 & $12.3 \pm 0.2$ & - & $11.9 \pm 0.1$ & - & - & $12.06 \pm 0.16$ \\
1,4 & $16.65 \pm 0.04$ & $16.58 \pm 0.07$ & $16.62 \pm 0.05$ & $16.61 \pm 0.05$ & $16.66 \pm 0.06$ & $16.62 \pm 0.05$ \\
2,2 & $5.10 \pm 0.17$ & $5.1 \pm 0.2$ & $4.71 \pm 0.08$ & $4.86 \pm 0.12$ & $4.90 \pm 0.14$ & $4.74 \pm 0.05$ \\
2,3 & $7.87 \pm 0.05$ & - & - & $7.87 \pm 0.06$ & $7.81 \pm 0.05$ & - \\
4,8 & $19.6 \pm 0.2$ & $19.17 \pm 0.08$ & $19.09 \pm 0.10$ & $19.8 \pm 0.4$ & $19.97 \pm 0.5$ & $19.08 \pm 0.10$ \\
4,12 & $34.86 \pm 0.05$ & $34.89 \pm 10$ & - & - & - & $34.90 \pm 0.07$ \\
6,14 & $33.67 \pm 0.05$ & - & $33.69 \pm 0.07$ & $33.62 \pm 0.06$ & $33.58 \pm 0.06$ & $33.68 \pm 0.07$ \\
6,15 & - & - & - & - & - \\
6,16 & $42.90 \pm 0.4$ & - & - & - & - & - \\
6,18 & - & - & $50.37 \pm 0.07$ & $50.16 \pm 0.08$ & $50.14 \pm 0.07$ & - \\
$\sigma(\mathrm{EMF}) / \mathrm{mV}$ & \pm 0.42 & \pm 0.70 & \pm 0.51 & \pm 0.51 & \pm 0.53 & \pm 0.49 \\
$U(\mathrm{EMF}) /(\mathrm{mV})^{2}$ & 17.27 & 45.03 & 25.85 & 27.80 & 27.55 & 23.86 \\
\hline
\end{tabular}

\section{Results and discussion}

The Th(IV) hydroxide complexes. Two of the mononuclear complexes, $\mathrm{ThOH}^{3+}$ and $\mathrm{Th}(\mathrm{OH})_{4}(\mathrm{aq})$, are well determined, and the equilibrium constants are not strongly dependent on the chemical model (cf. Table 2). However, $\mathrm{Th}(\mathrm{OH})_{4}(\mathrm{aq})$ makes a significant contribution only in experiments above $-\log \left[\mathrm{H}^{+}\right]=4$, and amounts to at most $15 \%$ of the total concentration of Th(IV). The values of $\log { }^{*} \beta_{1}$ and $\log { }^{*} \beta_{4}$ (and also $\log { }^{*} \beta_{3}$, even though this value is rather uncertain), indicate that the stepwise equilibrium constants for the reactions $\mathrm{Th}(\mathrm{OH})_{p}{ }^{4-p}+\mathrm{H}_{2} \mathrm{O} \rightleftharpoons$ $\mathrm{Th}(\mathrm{OH})_{p+1}{ }^{3-p}+\mathrm{H}^{+}$are approximately the same for $p=0-3$ $\left(\log { }^{*} K \approx-4\right)$. This is not the case for uranium(IV), the only M(IV) actinide for which information on mononuclear hydrolysis is available. ${ }^{5,14,15}$ Bruno et al. ${ }^{16}$ and Rai et al. ${ }^{17}$ have studied the hydrolysis of U(IV) by using solubility measurements. However, they arrive at very different conclusions on the stability of the mononuclear complexes. Both teams use an amorphous uranium(IV) hydroxide phase but find rather different solubilities; Bruno et al. have a minimum solubility of about $10^{-4} \mathrm{M}$, while Rai et al. report $10^{-8} \mathrm{M}$. This may be due to differences in the specific surface area and crystallinity, but also to difficulties in keeping uranium in the +IV state. The latter point is extensively discussed by Rai et al. Bruno et al. report equilibrium constants for $\mathrm{U}(\mathrm{OH})_{2}{ }^{2+}, \mathrm{U}(\mathrm{OH})_{3}{ }^{+}$and $\mathrm{U}(\mathrm{OH})_{4}$ (aq) that do not fit other uranium(IV) data, as discussed by the NEA review team. ${ }^{15}$ From the data of Rai et al. obtained at $21 \pm 2{ }^{\circ} \mathrm{C}$ one obtains equilibrium constants for reactions (1) and (2).

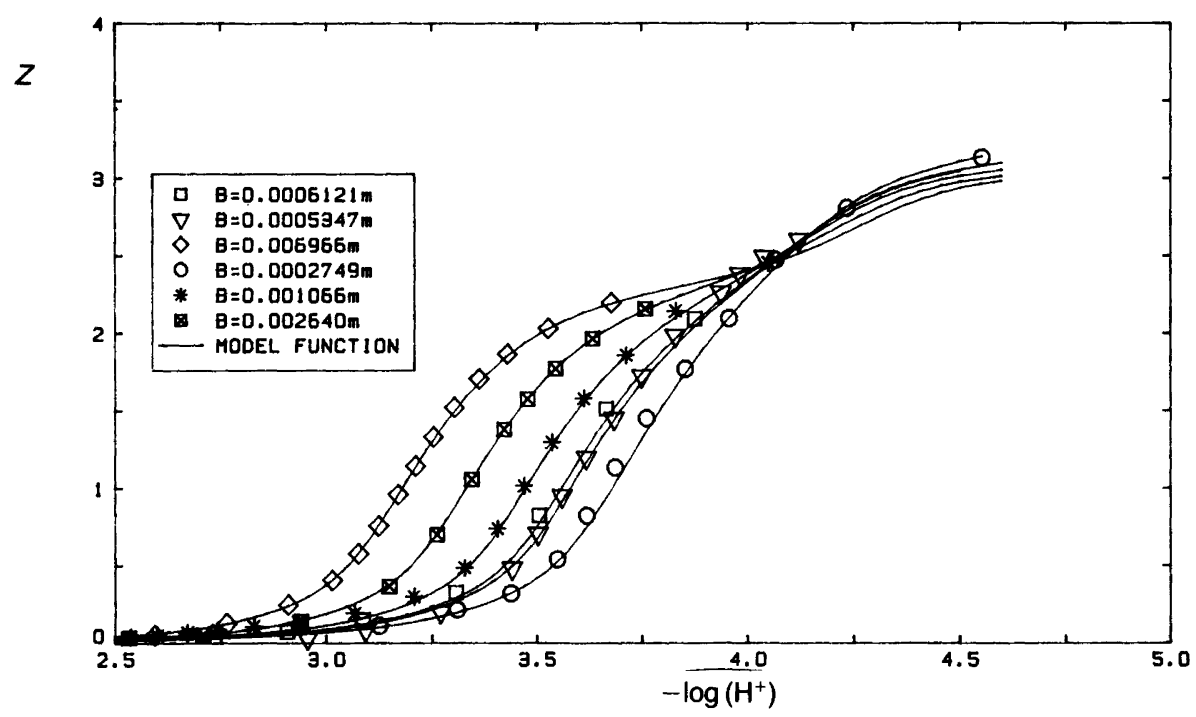

Fig. 1. Experimental data $Z\left(-\log \left[\mathrm{H}^{+}\right],[\mathrm{Th}]_{\text {tot }}=B\right)$ for the $\mathrm{Th}(\mathrm{IV})-\mathrm{H}_{2} \mathrm{O}$ system at $25^{\circ} \mathrm{C}$ in $3 \mathrm{M} \mathrm{NaClO}{ }_{4}$ where $Z=-\left(H_{\text {tot }}-\left[\mathrm{H}^{+}\right]\right) / B($ the average number of $\mathrm{OH}^{-}$bound per thorium) and $B=$ total concentration of Th(IV). The experimental points are indicated and the full curves are calculated by using the selected chemical model, model 1 of Table 3. 


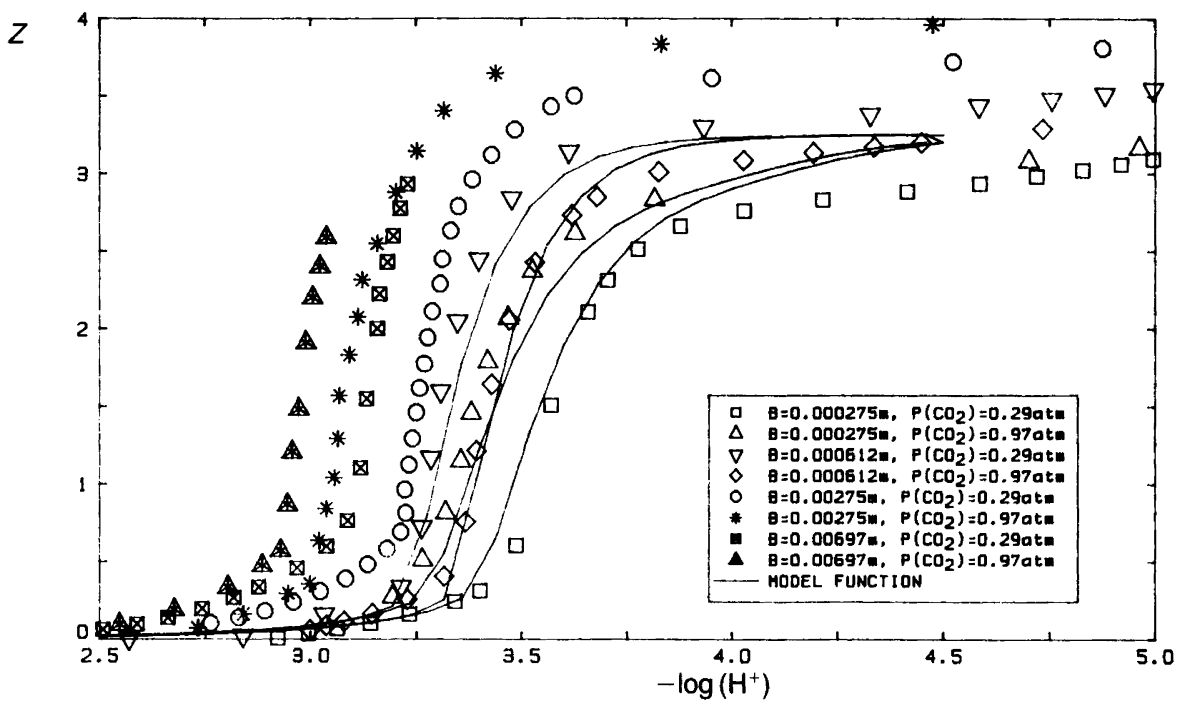

Fig. 2. Experimental data $Z\left(-\log \left[\mathrm{H}^{+}\right],[\mathrm{Th}]_{\mathrm{tot}}=B, P_{\mathrm{CO}_{2}}\right)$ for the $\mathrm{Th}(\mathrm{IV})-\mathrm{H}_{2} \mathrm{O}-\mathrm{CO}_{2}(\mathrm{~g})$ system at $25^{\circ} \mathrm{C}$ in $3 \mathrm{M} \mathrm{NaClO}$, where $Z=-\left(H_{\text {tot }}-\left[\mathrm{H}^{+}\right]+\left[\mathrm{HCO}_{3}{ }^{-}\right]+2\left[\mathrm{CO}_{3}{ }^{2-}\right]\right) / B$ (the average number of $\mathrm{OH}^{-}$bound per thorium) and $B=$ total concentration of Th(IV). The full curves are calculated by using a tentative model containing model 1 of Table 3 plus the complexes $\mathrm{Th}_{16}(\mathrm{OH})_{52}\left(\mathrm{CO}_{2}\right)_{16}{ }^{12+}$ and $\mathrm{Th}_{8}(\mathrm{OH})_{24}\left(\mathrm{CO}_{2}\right)_{2}^{8+}$.

$$
\begin{aligned}
& \mathrm{U}(\mathrm{OH})_{4}(\mathrm{~s}, \text { amorph })+3 \mathrm{H}^{+} \rightleftharpoons \mathrm{UOH}^{3+}+3 \mathrm{H}_{2} \mathrm{O} \log K^{\circ}=3.5 \\
& \mathrm{U}(\mathrm{OH})_{4}(\mathrm{~s}, \text { amorph }) \rightleftharpoons \mathrm{U}(\mathrm{OH})_{4}(\mathrm{aq}) \quad \log K^{\circ}=-8
\end{aligned}
$$

From the known equilibrium constant for reaction (3) we ob-

$$
\mathrm{U}^{4+}+\mathrm{H}_{2} \mathrm{O} \rightleftharpoons \mathrm{UOH}^{3+}+\mathrm{H}^{+} \quad \log * \beta_{1}^{\circ}=-0.5
$$

tain $\log * \beta_{4}^{\circ}=-11.5 \pm 1.0$ for reaction (4), as compared to

$$
\mathrm{U}^{4+}+4 \mathrm{H}_{2} \mathrm{O} \rightleftharpoons \mathrm{U}(\mathrm{OH})_{4}(\mathrm{aq})+4 \mathrm{H}^{+}
$$

$\log \beta_{4}^{\circ}=-14.3$ for the $T h(I V)$ system. It seems that the main difference between the Th(IV) and U(IV) hydrolysis complexes is found in the first hydrolysis constant. The same type of behaviour is also found in the corresponding mononuclear fluoride complexes. ${ }^{18}$

Ryan and Rai ${ }^{19}$ have studied the solubility of hydrous amorphous $\mathrm{ThO}_{2}$. The main conclusion of their study is that no anionic hydroxide complexes are formed, i.e. the same finding as in a previous study of the U(IV) system. ${ }^{17}$ The authors also discuss the formation of mononuclear complexes and conclude as to "mononuclear hydrolyzed $\mathrm{Th}$ ions not being dominant solution species at least up to $\mathrm{pH} 4.5$ ". This conclusion is in agreement with our observations. Ryan and Rai also use their solubility data, together with previously published information on Th hydrolysis, ${ }^{4,5}$ to estimate the solubility product of the solid $\mathrm{ThO}_{2}$ used, and the equilibrium constant for the formation of $\mathrm{Th}(\mathrm{OH})_{4}(\mathrm{aq})$. The two quantities are dependent on one another, and Ryan and Rai propose $\log K_{\mathrm{s}} \approx-46$ and $\log * \beta_{4} \leq-19.7$.

The discrepancy between the latter value and the value -14.3 proposed in this study is very large. One obvious reason for such a discrepancy is errors in experimental procedures, or in the interpretation of the experimental data. On the basis of available information we cannot resolve the issue. However, Ryan and $\mathrm{Rai}^{19}$ have not presented an analysis of how well their chemical model (solubility product + equilibrium constants) describes their experimental data. There is no analysis of the uncertainty in the solubility product, and finally there is no analysis of the experimental uncertainties in the $\mathrm{pH}$ measurements. The latter point is important in view of the low buffering capacity of the solutions. Our experiments in the presence of carbon dioxide shows that the complex formation curves are much steeper than in the absence of carbonate. In view of this it is also of importance to know if the tetraethylammonium hydroxide used by Ryan and Rai, ${ }^{19}$ contained carbonate impurities. We suggest that a better experimental procedure than that used by Ryan and Rai is either to use coulometry in a closed system to change the $\mathrm{pH}$, or to use buffers with weak complex formers, such as monochloroacetic acid/monochloroacetate. In the latter case one can keep the concentration of the chloroacetate ion constant and vary the concentration of acid.

If we use the minimum solubility of Ryan and $\mathrm{Rai}^{19}$ and our proposed value of $\log { }^{*} \beta_{4}^{\circ}$ we obtain $\log K_{\mathrm{s}}^{\circ}=-50.7$ for reaction (5), which as expected is much higher than the

$\mathrm{ThO}_{2}(\mathrm{~s}, \mathrm{amorph})+4 \mathrm{H}^{+} \rightleftharpoons \mathrm{Th}^{4+}+4 \mathrm{OH}^{-}$

value for the crystalline oxide $\left(\log K_{\mathrm{s}}^{\circ}=-54.14\right)$. However, the conflicting evidence between previous studies, including our own, and that of Ryan and $\mathrm{Ra}^{19}$ can only be resolved by performing a better designed solubility study.

In order to compare the numerical values of the equilibrium constants among our study and those of 
previous investigators, we have used the specific ion interaction theory ${ }^{15}$ to take differences in ionic medium and ionic strength into account. The equilibrium constant $\log { }^{*} \beta_{1}=-4.12$ reported to Baes and $\mathrm{Mesmer}^{4}$ in $1 \mathrm{~m}$ $\mathrm{NaClO}_{4}$ is consistent with the value found in this study; a recalculation to $I=3 \mathrm{M} \mathrm{NaClO}_{4}$ using $\Delta \varepsilon=-0.14$ from the corresponding $U(\mathrm{IV})$ system $^{14}$ gives $\log { }^{*} \beta_{1}=-4.07$. The value of $\log { }^{*} \beta_{1}=-2.98$ in $0.1 \mathrm{M} \mathrm{KNO}_{3}$ from Brown et al. ${ }^{5}$ is larger than expected. Baes and Mesmer have also estimated $\log * \beta_{1}^{\circ}=-3.20$, which is also in fair agreement with our estimate of $\log * \beta_{1}^{\circ}=-3.27$. These constants refer to the reference state at zero ionic strength.

A similar analysis of the values $\log { }^{*} \beta_{2,2}$ at $1 \mathrm{~m}$ and $3 \mathrm{M}$ $\mathrm{NaClO}_{4}$ gives a very uncertain estimate of the ion interaction coefficient $\varepsilon\left[\mathrm{Th}_{2}(\mathrm{OH})_{2}{ }^{6+}, \mathrm{ClO}_{4}{ }^{-}\right]=1.2 \pm 0.2$. This value has been calculated using the ion interaction coefficients selected by NEA. ${ }^{15}$ The complex $\mathrm{Th}_{2}(\mathrm{OH})_{3}{ }^{5+}$ has previously only been found in chloride and nitrate media. ${ }^{20,21}$ It is present in appreciable concentrations in this study, and one reason for its detection might be that we have used low total concentrations of Th(IV), which will promote the formation of complexes of low nuclearity in comparison with those of high.

The equilibrium constant for reaction (6), $\log { }^{*} K=-2.7$,

$$
\mathrm{Th}_{2}(\mathrm{OH})_{2}{ }^{6+}+\mathrm{H}_{2} \mathrm{O} \rightleftharpoons \mathrm{Th}_{2}(\mathrm{OH})_{3}{ }^{5+}+\mathrm{H}^{+}
$$

indicates that $\mathrm{Th}_{2}(\mathrm{OH})_{2}{ }^{6+}$ is a much stronger acid than $\mathrm{Th}^{4+}$, which makes chemical sense. The complexes $\mathrm{Th}_{4}(\mathrm{OH})_{8}{ }^{8+}$ and $\mathrm{Th}_{6}(\mathrm{OH})_{14}{ }^{10+}$ have been identified previously, and the corresponding equilibrium constants are in fair agreement with previous determinations. The equilibrium constant for the $(6,14)$ complex is not very dependent on the chemical model, while the value for the $(4,8)$ complex is; cf. Table 2 . For the ions of charge +8 or higher we have made no attempt to use the specific ion interaction theory. The $(6,16)$ complex is present in small amounts, and the existence of this species is by no means proven; however, its stoichiometry fits very well with the known ${ }^{6}$ geometry of the $(6,14)$ complex.

The complex $\mathrm{Th}_{4}(\mathrm{OH})_{12}{ }^{4+}$ was first proposed by Danesi et al. ${ }^{21}$ and it gives a better fit to our data than the $(6,18)$ complex. The $(6,18)$ complex has not been tested by Danesi et al.; if it is formed at all its formation should be promoted by the very high Th(IV) concentrations used by these authors. The $(4,12)$ stoichiometry is structurally quite feasible, and might be related to the known $(4,8)$ structure by adding terminal $\mathrm{OH}$ groups to each of the Th ions at the corners of the $\mathrm{Th}_{4}$ tetrahedron.

Thorium carbonate complexes. The experimental data indicate clearly that carbonate-containing thorium species are formed and that their nuclearity differs substantially from those of the hydroxide complexes. How are these species formed, and is there any relationship between the hydroxide and the carbonate species? To us, it seems most likely that the carbonate complexes are formed by the addition of $\mathrm{CO}_{2}$ to a coordinated oxide, forming a bridge linking several such units together. For example, we may imagine that $\mathrm{Th}_{8}(\mathrm{OH})_{24}\left(\mathrm{CO}_{2}\right)^{8+}$ is formed from $\mathrm{Th}_{4}(\mathrm{OH})_{12}{ }^{4+}$ by using a minimum of one bridging carbonate group. However, at this stage all discussions of the stoichiometry of these complexes will be speculative.

It is interesting to observe that U(IV) has properties similar to the Th(IV) system, but that the carbonate effect occurs at a $-\log \left[\mathrm{H}^{+}\right]$value about 1.5 units lower than for Th(IV). Our results indicate that the $M(I V)$ carbonate hydroxide complexes are quite stable. It would be interesting to study them in solutions containing fluoride, which forms more stable complexes than hydroxide, but which is also much less prone to the formation of polynuclear complexes. Such a system might be simpler to analyze because one can arrange the experimental conditions so that the hydroxide complexes are minor species.

Environmental consequences of carbonate complexes for the mobility of actinide(IV) ions. It seems quite obvious from the results obtained in this study that carbonate may also affect the speciation, and hence the solubility of actinide (IV) compounds, at $\mathrm{pH}<7$. The complexes formed have both a large size and a high charge, which may affect their diffusion and sorption properties.

Acknowledgements. This study has been supported by a grant from The Swedish Nuclear Fuel and Waste Management Co. (SKB). Dr. Marco Caceci is gratefully acknowledged for carrying out the dynamic light-scattering experiments. We also thank Dr. Jordi Bruno for fruitful discussions and a much encouragement throughout this work.

\section{References}

1. Ciavatta, L., Ferri, D., Salvatore, F. and Spahiu, K. Inorg. Chem. 22 (1983) 2088.

2. Bruno, J., Grenthe, I. and Robouch, P. Inorg. Chim. Acta 158 (1989) 221.

3. Bruno, J., Casas, I., Grenthe, I. and Lagerman, B. Inorg. Chim. Acta 140 (1987) 299.

4. Baes, C. F. and Mesmer, R. E. The Hydrolysis of Cations, Wiley, New York 1976, pp. 158-168.

5. Brown, P. L., Ellis, J. and Sylva, R. N. J. Chem. Soc., Dalton Trans. (1983) 31

6. Johansson, G. Acta Chem. Scand. 22 (1968) 389.

7. Magni, M., Cabrini, A., Scibona, G., Johansson, G. and Sandström, M. Acta Chem. Scand., Ser. A 30 (1976) 437.

8. Johansson, G. Acta Chem. Scand. 22 (1968) 399.

9. Bruno, J. J. Chem. Soc., Dalton Trans. (1987) 2431.

10. Vogel, A. I. Text-Book of Quantitative Inorganic Analysis, Including Elementary Instrumental Analysis, 3rd ed., Longman, London 1961, p. 540.

11. Gran, G. Calculation of Equivalence Volumes in Potentiometric Titrations, Thesis, Royal Institute of Technology, Stockholm, Sweden 1981, p. 25.

12. Ekelund, R., Sillén, L. G. and Wahlberg, O. Acta Chem. Scand. 24 (1970) 3073. 
13. Ahrland, S., Bagnall, K. W., Brown, D., Dell, R. M., Eberle, S. H., Keller, C., Lee, J. A., Liljenzin, J. O., Mardon, P. G., Marples, J. A. C., Milner, G. W. C., Phillips, G., Potter, P. E. and Rydberg, J. The Chemistry of the Actinides, Pergamon Texts in Inorganic Chemistry, 10. Pergamon Press, Oxford 1975 , p. 277.

14. Grenthe, I., Bidoglio, G. and Omenetto, N. Inorg. Chem. 28 (1989) 71

15. Grenthe, I., Fuger, J., Lemire, R. J., Muller, A. B., NguyenTrung, C. and Wanner, H. Chemical Thermodynamics of Uranium, Nuclear Energy Agency, OECD-NEA, Paris 1990. In press.

16. Bruno, J., Casas, I., Lagerman, B. and Munoz, M. Mater. Res. Soc. Symp. Proc. 84 (1987) 153.
17. Rai, D., Felmy, A. R. and Ryan, J. L. Inorg. Chem. 29 (1990) 260.

18. Ahrland, S., Hefter, G. and Norén, B. Acta Chem. Scand. 44 (1990) 1.

19. Ryan, J. L. and Rai, D., Inorg. Chem. 26 (1987) 4140.

20. Hietanen, S. and Sillén, L. G. Acta Chem. Scand. 22 (1968) 265.

20. Milič, N. B. Acta Chem. Scand. 25 (1971) 2487.

21. Danesi, P. R., Magini, Margherita, S. and D'Alessandro, Energ. Nucl. (Milan) 15 (1968) 335.

Received May 24, 1990. 